\title{
Serum miR-551b-3p is a potential diagnostic biomarker for gastric cancer
}

\author{
Su-yang Bai*1 (D), Rui Ji ${ }^{\star 1}$ (D), Hui Wei' (D), Qing-hong Guo ${ }^{1}$ (D), Hao Yuan ${ }^{1}$ (D), Zhao-feng Chen ${ }^{1}$ (D), Yu-ping Wang ${ }^{1}$ (D) Zheng Liu² (D), \\ Xiao-yan Yang ${ }^{2}$ (D), Yong-ning Zhou' ${ }^{\text {(D) }}$ \\ ${ }^{1}$ Department of Gastroenterology, The First Hospital of Lanzhou University; Key Laboratory for Gastrointestinal Diseases of Gansu Province, \\ Lanzhou, Gansu, China \\ ${ }^{2}$ The First Clinical Medical School of Lanzhou University, Lanzhou, Gansu, China
}

Cite this article as: Bai S, Ji R, Wei H, et al. Serum miR-551b-3p is a potential diagnostic biomarker for gastric cancer. Turk J Gastroenterol 2019; 30(5): 415-9.

\begin{abstract}
Background/Aims: Gastric cancer (GC) is one of the most common gastrointestinal malignancies. Many studies have demonstrated that serum microRNAs have potential applications as non-invasive biomarkers for cancer diagnosis. The aim of the present study was to investigate the expression of serum miR-551b-3p in patients with GC and to explore its potential as a diagnostic biomarker in GC. Materials and Methods: The expression of miR-551b-3p was detected using quantitative reverse transcription polymerase chain reaction in preoperative serum samples of 50 patients with GC and 53 healthy individuals. An analysis was performed to determine the correlation between serum miR-551b-3p levels and clinicopathological characteristics of patients with GC. The receiver operating characteristic curve was generated, and the cut-off point of serum miR-551b-3p for the diagnosis of GC was selected. The clinical value of serum miR-551b-3p for GC was analyzed by a consistency test.

Results: The expression of serum miR-551b-3p was significantly lower in patients with GC than in healthy individuals ( $p=0.000)$. Low level was positively associated with tumor size $(p=0.014)$, depth of invasion $(p=0.001)$, and Tumor-Node-Metastasis stage $(p=0.022)$. The area under the curve for serum miR-551b-3p distinguishing patients with GC from healthy individuals was 0.860 (95\% Cl: $0.787-$ $0.933, p=0.000$ ), with a specificity of $96.2 \%$ and a sensitivity of $70 \%$. The kappa consistency test had a kappa value of 0.667 ( $p=0.000)$ in GC.

Conclusion: Serum miR-551b-3p may potentially serve as a diagnostic biomarker for GC

Keywords: MicroRNAs, serum, biomarkers, stomach neoplasms
\end{abstract}

\section{INTRODUCTION}

Gastric cancer (GC) is one of the most common malignancies worldwide. It is an important cause of cancer-related death; its incidence ranks fourth among all malignant tumors worldwide, and the mortality rate ranks second in the world (1). Most GC-related deaths can be prevented through early diagnosis and surgical removal of the early tumor. Thus, effective early screening and diagnosis are keys to increasing the survival rate of patients with gastric carcinoma, but invasive gastroscopy is unpopular with patients. The specificity and sensitivity of clinical carcinoembryonic antigen, carbohydrate antigen 19-9, carbohydrate antigen $72-4$, and other serum biomarkers are not sufficient for the diagnosis of GC. Therefore, there is an urgent need for new effective biomarkers to promote the detection of GC in clinical practice.

Recent studies have demonstrated that microRNAs (miRNAs), a class of highly conserved, small, non-coding
RNAs, are abnormally expressed in a variety of tumor tissues and remain stable in the blood (2). These features suggest that serum miRNAs can be molecular tools for detecting and monitoring various types of cancer (3).

In the early stage, miRNA microarray results showed that 30 miRNAs were significantly differentially expressed in GC tissues compared with adjacent normal tissues. Our research group selected miR-551b-3p from 30 miRNAs and confirmed that miR-551b-3p showed low expression in GC by molecular biology techniques (4). However, the expression level of miR-551b-3p in the serum of a patient with GC remains unclear. In the present study, real-time fluorescence quantitative polymerase chain reaction (qPCR) was used to evaluate the level of miR$551 b-3 p$ in the serum of patients with GC. Furthermore, we investigated the correlation between its expression and clinicopathological characteristics and explored its potential as a diagnostic biomarker of GC.

*These authors contributed equally to the study.

Corresponding Author: Yong-ning Zhou; yongningzhou@sina.com

Received: January 4, 2018 Accepted: October 1, 2018 Available online date: April 8, 2019

(C) Copyright 2019 by The Turkish Society of Gastroenterology · Available online at www.turkjgastroenterol.org

DOI: $10.5152 /$ tjg.2019.17875 


\section{MATERIALS AND METHODS}

\section{Specimen collection}

A total of 103 serum samples were collected in the present study, with $50 \mathrm{GC}$ samples and 53 normal control samples. All GC samples were obtained from preoperative fasting venous blood of patients with GC who had been confirmed by pathology in the oncology Department of Gastroenterology, The First Hospital of Lanzhou University. All normal control specimens were collected from healthy individuals who had no tumor history, no precancerous lesion, and normal biochemical indexes at the Medical Center of Lanzhou University. All subjects filled out a questionnaire. Informed consent was obtained from the patients to participate in the study. The ethics committee of Lanzhou University approved the study.

\section{Serum RNA extraction and RT-qPCR}

Total RNA was extracted from $300 \mu$ l serum using TRIzol reagent (Invitrogen, Carlsbad, CA, USA) according to the manufacturer's protocol. Then, the RNA was reverse transcribed using TIANScript M-MLV (Tiangen Biotech Beijing Co., Ltd., Beijing, China). hsa-miRNA-16 (miR-16) was used as an internal reference in the present study. qPCR to detect the expression levels of miR-551b-3p was performed using a LightCycler PCR System (Roche, Mannheim, Germany) with a $10 \mu$ l quantitative reverse transcription polymerase chain reaction (RT-qPCR) reaction mixture, comprising forward primer $(0.5 \mu \mathrm{l})$, reverse primer $(0.5 \mu \mathrm{l})$, cDNA $(2 \mu \mathrm{l})$, SYBR Premix Ex Taq II (Tli RNase H Plus, $2 \times)(5 \mu l)$, and RNase/DNase-free water (2 $\mu l)$. The reaction was incubated in a 96 -well plate at $95^{\circ} \mathrm{C}$ for $30 \mathrm{~s}$, followed by 50 cycles of $95^{\circ} \mathrm{C}$ for $5 \mathrm{~s}$ and $60{ }^{\circ} \mathrm{C}$ for $30 \mathrm{~s}$. The relative expression of serum miR-551b-3p was calculated using the $2^{-\Delta C t}$ method. The results were normalized to the expression of miR-16. Table 1 shows the primers used in the present study.

\section{Statistical analysis}

All statistical analyses and graphics were performed using Statistical Package for Social Sciences version 24.0 (IBM Corp.; Armonk, NY, USA) and GraphPad Prism 5.0 (GraphPad Software, San Diego, CA, USA). Values are expressed as median (lower quartile and upper quartile). The Mann-Whitney $U$ tests were used to compare the serum miR-551b-3p levels between patients with GC and healthy controls. Analyses of the miR-551b-3p expression levels and the clinicopathological parameters in GC were performed using the Mann-Whitney $U$ tests. The diagnostic efficacy of serum miR-551b-3p as a biomarker was assessed by the receiver operating characteristic
(ROC) curve analysis and the kappa consistency test. A p value $<0.05$ was considered statistically significant.

\section{RESULTS}

\section{Serum miR-551b-3p is downregulated in the GC group compared with healthy controls}

We detected the expression status of miR-551b-3p in 50 patients with GC and 53 healthy controls using RT-qPCR. As shown in Figure 1, the results indicated that compared with healthy controls, the GC group showed significantly downregulated levels of serum miR-551b-3p $(p=0.000)$.

\section{Clinical significance of serum miR-551b-3p expression level in the GC group}

The serum miR-551b-3p expression status of 50 patients with GC was analyzed prior to surgery. Table 2 shows the serum miR-551b-3p levels along with the clinicopathological features of patients with GC. As summarized in

Table 1. The primer sequence of serum miR-551b-3p and miR-16

Genes Primer sequences

miR-551b-3p

Forward 5'-ACACTCCAGCTGGGGCGACCCATACTTGG-3

Reverse 5'-ACTGGTGTCGTGGAGTCG-3'

miR-16

Forward 5'-ACACTCCAGCTGGGTAGCAGCACGTAAATA-3'

Reverse 5'-ACTGGTGTCGTGGAGTCG-3'

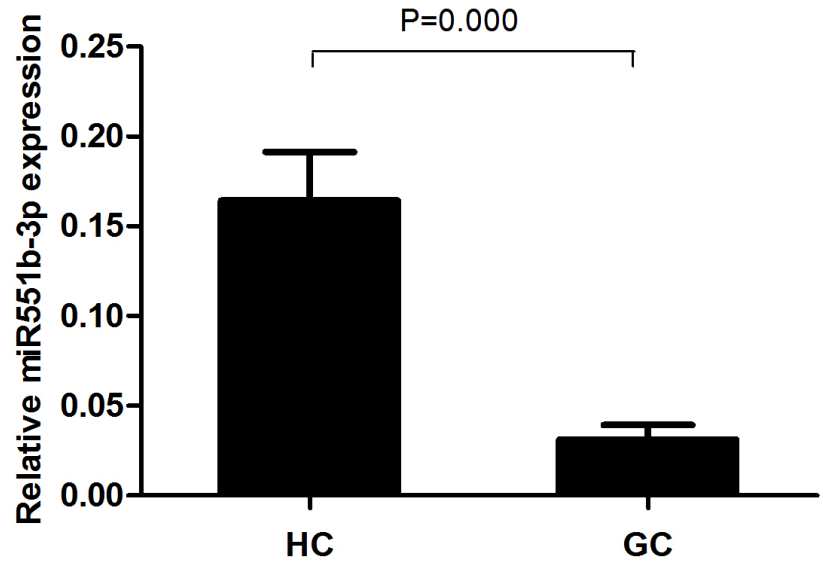

Figure 1. The relative expression of serum miR-551b-3p in the gastric cancer group and healthy control group HC: healthy control; GC: gastric cancer 


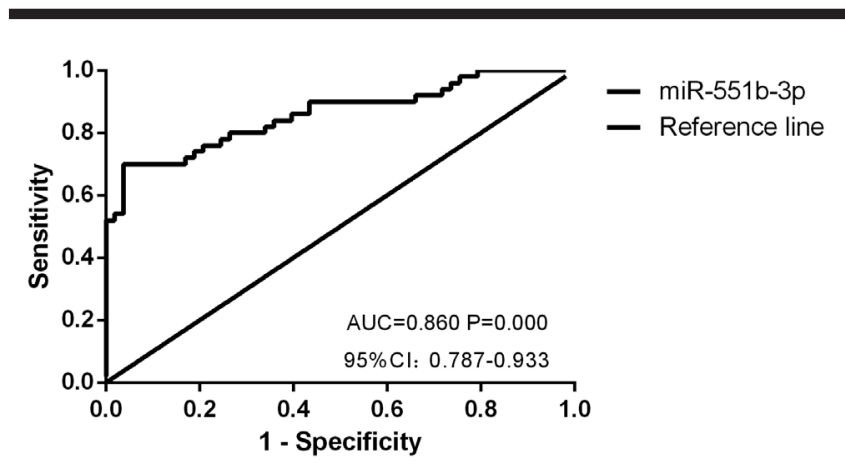

Figure 2. Diagnostic value of serum miR-551b-3p expression for gastric cancer patients

Table 2, the serum levels of $\mathrm{miR}-551 \mathrm{~b}-3 \mathrm{p}$ were significantly correlated with tumor size $(p=0.014)$, depth of invasion $(p=0.001)$, and Tumor-Node-Metastasis (TNM) stage $(p=0.022)$. However, there was no association between serum expression levels of miR-551b-3p and other clinicopathological features, including age, gender, histological grade, and lymph node metastasis ( $p>0.05)$.

\section{Diagnostic accuracy of serum miR-551b-3p for GC}

For evaluation of the diagnostic accuracy of serum miR$551 b-3 p$ as a GC biomarker, we generated the ROC curve of miR-551b-3p in the GC group and healthy control group. As shown in Figure 2, the area under the ROC curve was 0.860 (95\% Cl: $0.787-0.933, p=0.000)$. The sensitivity and specificity of this method were $70 \%$ and $96.2 \%$, respectively. The Youden index was 0.662 , and the cut-off point was 0.0175 . The ROC curve analysis demonstrated that serum miR-551b-3p was a potential biomarker for screening patients with GC.

\section{Clinical application value of serum miR-551b-3p in the diagnosis of GC}

For assessment of the clinical value of serum miR-551b$3 p$ in the diagnosis of GC, we performed a kappa consistency analysis of the serum miR-551b-3p diagnosis and pathological diagnosis for GC. Using pathological diagnosis as the gold standard for cancer, we obtained the cut-off point (0.0175) of serum miR-551b-3p with ROC curve analysis (Figure 2). As indicated in Table 3, GC was a positive result, and non-cancer was a negative result. The test results showed that the diagnostic sensitivity and specificity of serum miR-551b-3p were $70 \%$ and $96.2 \%$, respectively. The misdiagnosis (a), omission diagnostic $(\beta)$, diagnostic coincidence $(\pi)$, positive predictive value $\left(\mathrm{PV}_{+}\right)$, negative predictive value $\left(\mathrm{PV}_{-}\right)$, and consistency test kappa rates were 3.8\%, 30\%, 83.5\%, 94.6\%,
Table 2. Correlations of serum miR-551b-3p expression levels and clinicopathological parameters of patients with gastric cancer

\begin{tabular}{lcc}
$\begin{array}{l}\text { Clinical } \\
\text { parameters }\end{array}$ & $\begin{array}{c}\text { Case } \\
(\mathrm{n}=50)\end{array}$ & $\begin{array}{c}\text { Expression levels of } \\
\text { serum miR-551b-3 }\end{array}$ \\
\hline $\begin{array}{l}\text { Age (years) } \\
\geq 60\end{array}$ & 22 & $\begin{array}{c}0.0061 \\
(0.0013,0.0251)\end{array}$ \\
$<60$ & 28 & $\begin{array}{c}0.0067 \\
(0.0012,0.0306)\end{array}$ \\
Gender & & \\
Male & 37 & 0.0047 \\
& & $(0.0005,0.0198)$ \\
Female & 13 & 0.0168 \\
& & $(0.0060,0.0444)$
\end{tabular}

Tumor size $(\mathrm{cm})$ $\geq 5$

$<5$

24

26

Histological grade

Well and moderate

Poor and others

Depth of invasion

$\mathrm{T} 1$ and $\mathrm{T} 2$

T3 and T4

Lymph node metastasis

Present

23

Absent

27

TNM stage

I and II

28

III and IV

30

22
0.05 0.755

0.014

0.0129

(0.0041, 0.0551)

0.0028

$(0.0003,0.0084)$

0.349

0.0020

$(0.0006,0.0266)$

$43 \quad 0.0071$

$(0.0015,0.0320)$

0.001

0.0016

$(0.0002,0.0063)$

0.0113

$(0.0045,0.0571)$

0.117

0.0076

$(0.0035,0.0568)$

0.0052

$(0.0004,0.0266)$

0.022

0.0020

(0.0003, 0.0228)

0.0078

$(0.0045,0.0604)$
TNM: Tumor-Node-Metastasis 
Table 3. Comparison of serum miR-551b-3p diagnosis for gastric cancer and pathological diagnosis

\begin{tabular}{lcccc}
\hline & & \multicolumn{2}{c}{ Pathological diagnosis } & \multirow{2}{*}{ Total } \\
\cline { 3 - 4 } & & Positive & Negative & \\
\hline miR-551b-3p & Positive & 35 & 2 & 37 \\
diagnosis & Negative & 15 & 51 & 66 \\
& & 50 & 53 & 103 \\
\hline
\end{tabular}

$77.3 \%$, and 0.667 , respectively $(p=0.000,<0.0001)$. These data indicated that the consistency of the two diagnostic methods was good.

\section{DISCUSSION}

Owing to the ease of sample collection, rapid detection, and patient acceptance, detection of serum tumor markers has been a good tool for diagnosis and screening of cancer, evaluation of curative effect, and patient prognosis. Recently, miRNAs, which are widely found in multicellular organisms and participate in various biological processes, have attracted increasing attention owing to their abnormal expression in many tumor tissues and tumor cells. Multiple studies have confirmed that miRNAs are stable in various body fluids, such as blood, urine, and saliva, and can be stored in vitro for a long time without degradation $(2,5)$. Therefore, miRNAs have obvious advantages as tumor markers (6-8).

In recent years, researchers have performed multiple studies on the application of miRNAs in the diagnosis and treatment of cancer and prognosis. A number of studies have shown that the abnormal expression of serum miR21 is closely related to the development of various tumors $(9-12)$. In the past few years, the abnormal expression of many miRNAs was detected in the serum of patients with non-small cell lung cancer $(5,13-17)$, prostate cancer $(2,18,19)$, breast cancer (20-23), and colorectal cancer $(24,25)$. These miRNAs all had potential as ideal tumor markers. Moreover, there were many studies about serum miRNAs in GC. Liu et al. (26) identified the serum expression of miR-1, miR-20a, miR-27a, miR-34, and miR-423$5 p$ in GC. Liu et al. (27) confirmed that miR-378 is highly expressed in the serum of patients with $\mathrm{GC}$ and can be used as an early screening marker for GC. Recently, the aberrant expression of serum miR-17 (28), miR-106b (28), miR-107 (29), miR-200c (30), miR-203 (31), miR-204 (32), miR-503 (33), miR-21 (12), and miR-331 (12) was confirmed to be closely related to GC, and these molecules may be candidate serum diagnostic markers for GC.
The aim of the present study was to evaluate the serum miR-551b-3p levels of patients with GC and to explore its potential as a diagnostic marker for GC. The results showed that serum miR-551b-3p expression decreased markedly in patients with GC compared with normal controls. Analysis of the correlations between serum miR551b-3p expression and clinicopathological features of GC indicated that there were no correlations between serum miR-551b-3p expression and age, gender, tumor differentiation, and lymph node metastasis, but the correlations with tumor size, depth of invasion, and TNM stage were statistically significant. The optimal diagnostic cut-off value (0.0175) of serum miR-551b-3p obtained by ROC analysis defined the positive and negative results. In addition, we compared the serum miR-551b-3p diagnosis results with the pathological diagnosis results using a consistency test. The results showed that the sensitivity of serum miR-551b-3p diagnosis of GC was $70 \%$, specificity was $96.2 \%$, the diagnostic coincidence rate was $83.5 \%$, PV , was $94.6 \%$, PV _ was $77.3 \%$, the kappa value was 0.667 , and $p=0.000(p<0.0001)$. The diagnostic consistency of the two methods was good. In summary, miR-551b-3p may be a new candidate biomarker for GC.

There were some limitations in the present study. These include the small number of samples and specimens from the same area. The overall results were statistically significant, but whether miR-551b-3p can be used as a reliable diagnostic indicator of GC and can be used clinically requires further research and verification. These findings should be confirmed and evaluated in prospective, large-sample, multicenter, randomized clinical trials.

Ethics Committee Approval: Ethics committee approval was obtained for this study from the Ethics Committee of Lanzhou University.

Informed Consent: Written informed consent was obtained from the patients who participated in this study.

Peer-review: Externally peer-reviewed.

Author Contributions: Concept - Y.Z., S.B., R.J., H.Y., Z.C.; Design S.B., R.J., H.W.; Supervision - S.B., R.J., Y.W., Y.Z.; Resources Y.Z., Y.W.; Materials - Y.Z., Z.C.; Data Collection and/or Processing - S.B., H.W., Q.G., H.Y., Z.L., X.Y.; Analysis and/or Interpretation - S.B., R.J., H.Y., Z.L., X.Y., Q.G.; Literature Search - H.W., Z.L., X.Y.; Writing Manuscript - S.B., R.J.; Critical Review - Q.G., Y.W., Z.C., Y.Z.

Conflict of Interest: The authors have no conflict of interest to declare.

Financial Disclosure: The authors declared that this study has received no financial support. 


\section{REFERENCES}

1. Chen W, Zheng R, Baade PD, et al. Cancer statistics in China, 2015. CA Cancer J Clin 2016; 66: 115-32. [CrossRef]

2. Mitchell $P$, Parkin R, Kroh E, et al. Circulating microRNAs as stable blood-based markers for cancer detection. Proc Natl Acad Sci USA 2008; 105: 10513-8. [CrossRef]

3. Cho W. Circulating MicroRNAs as Minimally Invasive Biomarkers for Cancer Theragnosis and Prognosis. Front Genet 2011; 2: 7. [CrossRef] 4. Chen Z, Liu X, Liu M, et al. [Expression of miR-551b-3p in gastric cancer cell lines and tissues and its clinical significance]. Zhonghua Zhong Liu Za Zhi 2014; 36: 903-4.

5. Chen $X, B a Y, M a L$, et al. Characterization of microRNAs in serum: a novel class of biomarkers for diagnosis of cancer and other diseases. Cell Res 2008; 18: 997-1006. [CrossRef]

6. Brase J, Wuttig D, Kuner R, Sültmann H. Serum microRNAs as non-invasive biomarkers for cancer. Mol Cancer 2010; 9: 306. [CrossRef]

7. Wittmann J, Jäck H. Serum microRNAs as powerful cancer biomarkers. Biochim Biophys Acta 2010; 1806: 200-7. [CrossRef]

8. Kosaka N, Iguchi H, Ochiya T. Circulating microRNA in body fluid: a new potential biomarker for cancer diagnosis and prognosis. Cancer Sci 2010; 101: 2087-92. [CrossRef]

9. Zhang $H$, Yang $L$, Zhu $Y$, et al. Serum miRNA-21: elevated levels in patients with metastatic hormone-refractory prostate cancer and potential predictive factor for the efficacy of docetaxel-based chemotherapy. Prostate 2011; 71: 326-31. [CrossRef]

10. Asaga $S$, Kuo $C$, Nguyen T, Terpenning M, Giuliano A, Hoon D. Direct serum assay for microRNA-21 concentrations in early and advanced breast cancer. Clin Chem 2011; 57: 84-91. [CrossRef]

11. Wang J, Huang $S$, Zhao $M$, et al. Identification of a circulating microRNA signature for colorectal cancer detection. PLOS ONE 2014; 9: e87451. [CrossRef]

12. Sierzega M, Kaczor M, Kolodziejczyk P, Kulig J, Sanak M, Richter $P$. Evaluation of serum microRNA biomarkers for gastric cancer based on blood and tissue pools profiling: the importance of miR-21 and miR-331. Br J Cancer 2017; 117: 266-73. [CrossRef]

13. Chen $X, H u Z$, Wang $W$, et al. Identification of ten serum microRNAs from a genome-wide serum microRNA expression profile as novel noninvasive biomarkers for nonsmall cell lung cancer diagnosis. Int J Cancer 2012; 130: 1620-8. [CrossRef]

14. Heegaard N, Schetter A, Welsh J, Yoneda M, Bowman E, Harris C. Circulating micro-RNA expression profiles in early stage nonsmall cell lung cancer. Int J Cancer 2012; 130: 1378-86. [CrossRef]

15. Foss K, Sima C, Ugolini D, Neri M, Allen K, Weiss G. miR-1254 and miR-574-5p: serum-based microRNA biomarkers for early-stage non-small cell lung cancer. J Thorac Oncol 2011; 6: 482-8. [CrossRef] 16. Bianchi F, Nicassio F, Marzi $M$, et al. A serum circulating miRNA diagnostic test to identify asymptomatic high-risk individuals with early stage lung cancer. EMBO Mol Med 2011; 3: 495-503. [CrossRef] 17. Yuxia M, Zhennan T, Wei Z. Circulating miR-125b is a novel biomarker for screening non-small-cell lung cancer and predicts poor prognosis. J Cancer Res Clin Oncol 2012; 138: 2045-50. [CrossRef]
18. Nguyen $H$, Xie $W$, Yang $M$, et al. Expression differences of circulating microRNAs in metastatic castration resistant prostate cancer and low-risk, localized prostate cancer. Prostate 2013; 73: 346-54. [CrossRef]

19. Selth L, Townley S, Bert A, et al. Circulating microRNAs predict biochemical recurrence in prostate cancer patients. Br J Cancer 2013; 109: 641-50. [CrossRef]

20. Sun $Y$, Wang $M$, Lin $G$, et al. Serum microRNA-155 as a potential biomarker to track disease in breast cancer. PLoS ONE 2012; 7 : e47003. [CrossRef]

21. Roth $C$, Rack B, Müller V, Janni W, Pantel K, Schwarzenbach $H$. Circulating microRNAs as blood-based markers for patients with primary and metastatic breast cancer. Breast Cancer Res 2010; 12 : R90. [CrossRef]

22. Sochor M, Basova P, Pesta M, et al. Oncogenic microRNAs: miR155, miR-19a, miR-181b, and miR-24 enable monitoring of early breast cancer in serum. BMC Cancer 2014; 14: 448. [CrossRef]

23. Chan M, Liaw C, Ji S, et al. Identification of circulating microRNA signatures for breast cancer detection. Clin Cancer Res 2013; 19: 4477-87. [CrossRef]

24. Ogata-Kawata $H$, Izumiya $M$, Kurioka $D$, et al. Circulating exosomal microRNAs as biomarkers of colon cancer. PLoS ONE 2014; 9 : e92921. [CrossRef]

25. Toiyama Y, Hur K, Tanaka K, et al. Serum miR-200c is a novel prognostic and metastasis-predictive biomarker in patients with colorectal cancer. Ann Surg 2014; 259: 735-43. [CrossRef]

26. Liu R, Zhang $\mathrm{C}, \mathrm{Hu} Z$, et al. A five-microRNA signature identified from genome-wide serum microRNA expression profiling serves as a fingerprint for gastric cancer diagnosis. Eur J Cancer 2011; 47: 78491. [CrossRef]

27. Liu H, Zhu L, Liu B, et al. Genome-wide microRNA profiles identify miR-378 as a serum biomarker for early detection of gastric cancer. Cancer Lett 2012; 316: 196-203. [CrossRef]

28. Zeng $Q$, Jin $C, C$ hen $W$, et al. Downregulation of serum miR-17 and miR-106b levels in gastric cancer and benign gastric diseases. Chin J Cancer Res 2014; 26: 711-6.

29. Ayremlou N, Mozdarani H, Mowla S, Delavari A. Increased levels of serum and tissue miR-107 in human gastric cancer: Correlation with tumor hypoxia. Cancer Biomark 2015; 15: 851-60. [CrossRef] 30. Zhang H, Sun F, Li S. Serum miR-200c expression level as a prognostic biomarker for gastric cancer. Genet Mol Res 2015; 14: 1591320. [CrossRef]

31. Imaoka H, Toiyama $Y$, Okigami $M$, et al. Circulating microR$N A-203$ predicts metastases, early recurrence, and poor prognosis in human gastric cancer. Gastric Cancer 2016; 19: 744-53. [CrossRef] 32. Chen X, Liu X, Liu H, Lu Y, Li Y. Reduced expression of serum miR204 predicts poor prognosis of gastric cancer. Genet Mol Res 2016; 15: gmr.15027702.

33. Wu D, Cao G, Huang Z, et al. Decreased miR-503 expression in gastric cancer is inversely correlated with serum carcinoembryonic antigen and acts as a potential prognostic and diagnostic biomarker. Onco Targets Ther 2017; 10: 129-35. [CrossRef] 